\title{
Intrinsic Dissolution as a Tool for Evaluating Drug Solubility in Accordance with the Biopharmaceutics Classification System
}

e-mail:sferraz@usp.br

\author{
Michele G. Issa and Humberto G. Ferraz* \\ Pharmacy Department, Pharmaceutical Sciences School, University of São Paulo, São Paulo, Brazil
}

\begin{abstract}
The Biopharmaceutics Classification System (BCS) is a tool that was created to categorize drugs into different groups according to their solubility and permeability characteristics. Through a combination of these factors and physiological parameters, it is possible to understand the absorption behavior of a drug in the gastrointestinal tract, thus contributing to cost and time reductions in drug development, as well as reducing exposure of human subjects during in vivo trials. Solubility is attained by determining the equilibrium under conditions of physiological $\mathrm{pH}$, while different methods may be employed for evaluating permeability. On the other hand, the intrinsic dissolution rate (IDR), which is defined as the rate of dissolution of a pure substance under constant temperature, $\mathrm{pH}$, and surface area conditions, among others, may present greater correlation to the in vivo dissolution dynamic than the solubility test. The purpose of this work is to discuss the intrinsic dissolution test as a tool for determining the solubility of drugs within the scope of the Biopharmaceutics Classification System (BCS).
\end{abstract}

\section{INTRODUCTION}

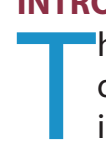

he bioavailability of a certain drug contained in a dosage form, which depends on a series of factors, including the physicochemical characteristics of its formulation and the physiological state of the patient's gastrointestinal tract, is very important for successful treatment (1). Amon $g$ the relevant characteristics, several properties relating to the absorption process are worth emphasizing: biopharmaceutical (solubility, dissolution, chemical stability, permeability, and first-pass effect), pharmacokinetic (renal clearance, biological half-life, protein binding, and distribution volume), pharmaceutical (formulation and dosage form), and physiological ( $\mathrm{pH}$, enzymes, intestinal motility, and i lity), which control the rate and the extent to which the drug reaches the site of action (2-4).

Many pharmaceutical companies have taken into account the properties of solubility and permeability, as well as physicochemical parameters, when undertaking the development of new drug candidates. This kind of evaluation is known as screening, which takes place in the initial phase of development and has helped to reduce the number of new molecules to be dealt with in the following stages, thus resulting in significant cost reductions $(3,5,6)$.

In 1995 the Biopharmaceutics Classification System (BCS) was proposed by Amidon and collaborators (7) to facilitate this work. This system considers that the dissolution and intestinal permeability of the active substance are the fundamental parameters in the rate and extent of absorption. Drugs are organized into four classes according to their solubility and permeability characteristics (7-10).

${ }^{*}$ Corresponding author.
According to the BCS, different methods can be used to evaluate solubility, intestinal permeability, and in vitro dissolution in the classification of drugs, and several of them are recommended by the $\operatorname{FDA}(9,11)$.

To determine the solubility of a drug, an evaluation of solubility equilibrium under conditions of physiological $\mathrm{pH}$ is recommended; in other words, this value should be determined at a temperature of $37 \pm 1^{\circ} \mathrm{C}$ in different solutions and $\mathrm{pH}$ values from 1 to 7.5 . The solubility class is obtained by calculating the volume of solution necessary to dissolve the greatest dose of the medication over all $\mathrm{pH}$ ranges evaluated (11). The method used is phase solubility, where an excess amount of the drug is placed in direct contact with the solution. However, it is worth remembering that in the initial stages of development, generally in the preformulation stage, the quantity of available material is often restricted, and so the execution of this study may not be feasible $(12,13)$.

Another point to be considered is the difficulty in correctly determining solubility, whether through experimental variables such as temperature variation, filtration, and quantity of material or factors inherent to the substance itself, which can affect the result and generate considerable data dispersion. Among these factors, alterations in the crystalline structure (solvates, hydrates, and polymorphs) or the formation of salts during the test can be cited $(12,14,15)$.

One alternative for determining solubility that has been discussed in recent years is the intrinsic dissolution test, where the solubility class of a drug is determined by the value of the intrinsic dissolution rate (IDR). The quantity of material required to carry out this test is much smaller than that required for the solubility test, and possible interference in the results by factors such as transition 
from crystalline structure or the formation of salts can be ascertained by the presence of curvatures in the graphs obtained (13, 15-19).

Thus, the purpose of this review is to discuss the use of the intrinsic dissolution test for evaluating the solubility of drugs within the context of the Biopharmaceutics Classification System.

\section{BIOPHARMACEUTICS CLASSIFICATION SYSTEM (BCS)}

The Biopharmaceutics Classification System was developed to promote the use of scientific knowledge in the evaluation of active substances, such as prediction of in vivo behavior, thus aiding regulatory agencies in approving new medicines and the pharmaceutical industry in obtaining product registrations (8). The main purpose of the BCS is to create a regulatory tool to substitute several bioequivalence tests for in vitro dissolution tests, thus enabling cost and time reductions in the development process of medications, as well as unnecessary exposure of human subjects during in vivo studies (8). Currently, the BCS is employed as a biowaiver for clinical bioequivalence tests involving orally administered formulations, especially those of immediate release, while its use is still under analysis for modified-release formulations $(8,9,20)$.

It is important to consider that a clinical response resulting from the oral administration of a certain medication will only occur after a series of events, thus constituting a complex process that can be influenced by factors related to the individual patient $(21,22)$. As can be observed in Figure 1, dissolution is the prerequisite for the absorption of orally administered drugs and consequently for a clinical response. Because dissolution depends on the solubility of the drug and absorption depends on intestinal permeability, these are the main factors that govern the rate and extent of absorption of an orally administered solid formulation, in other words, its bioavailability $(6,7,9,11)$.

Three factors represent the absorption behavior of a drug through the gastrointestinal tract $(6,7,9,10)$. Dose number $\left(D_{0}\right)$ is the dose concentration/solubility ratio

$$
D_{0}=\frac{M / V_{0}}{C_{s}}
$$

where $M$ is the dose, $V_{0}$ is the volume of water ingested during administration of the medication $(250 \mathrm{~mL})$, and $C_{\mathrm{s}}$ is the solubility. Dissolution number $\left(D_{n}\right)$ is the time required for dissolution

$$
D_{n}=\left(\frac{3 D}{r^{2}}\right)\left(\frac{C_{s}}{\rho}\right)\langle T s i\rangle=\frac{\left\langle T_{s i}\right\rangle}{T_{\text {diss }}}
$$

where $D$ is the diffusion coefficient, $r$ is the initial drug-particle radius, $C_{\mathrm{s}}$ is solubility, $\rho$ is density, $T_{\mathrm{si}}$ is intestinal transit time, and $T_{\text {diss }}$ is dissolution time. Absorption number $\left(A_{n}\right)$ is the time required to absorb the administered dose

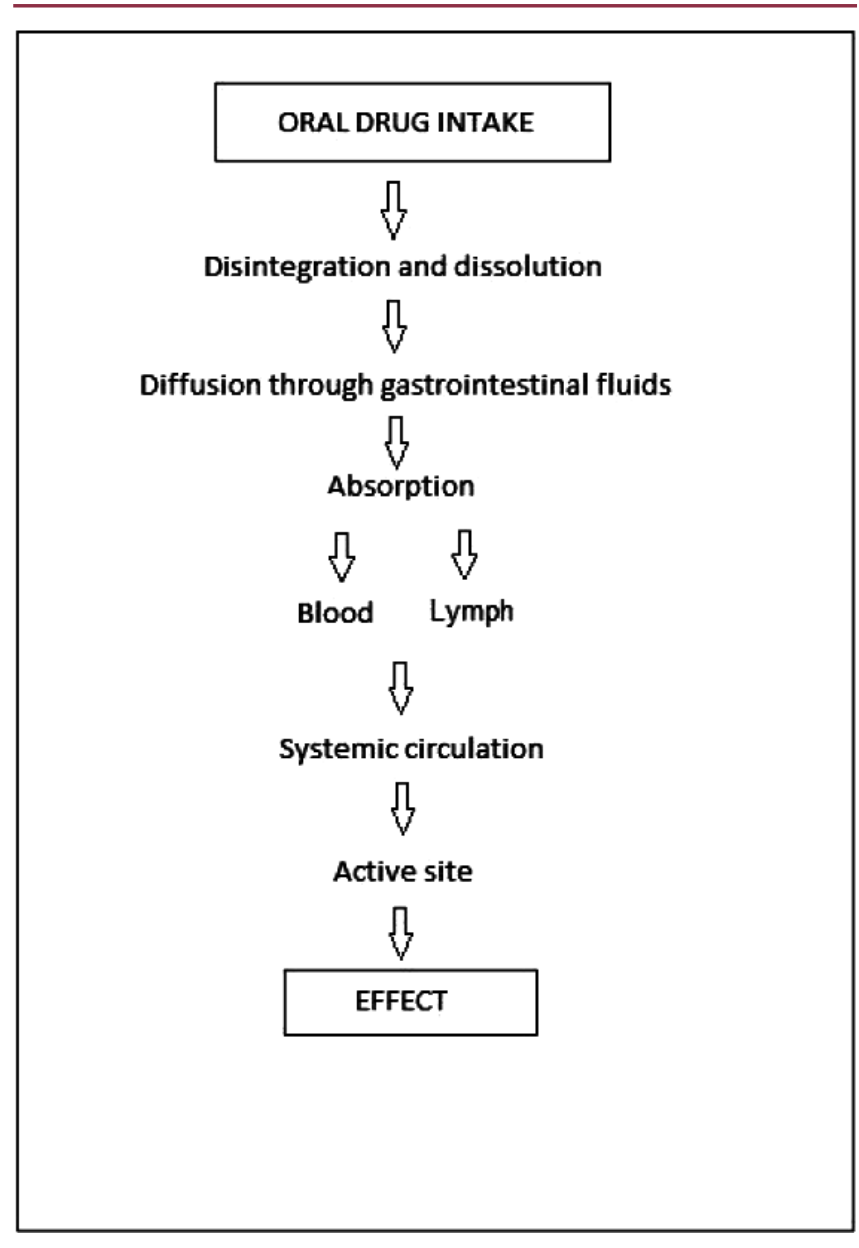

Figure 1. Sequence of events that take place from administration of medication to the action of the drug. (Adapted from ref 22. Copyright 2002 American College of Clinical Pharmacology, Inc.)

$$
A_{n}=\frac{P_{\text {eff }}}{R} x\langle T s i\rangle=\frac{\left\langle T_{s i}\right\rangle}{T_{a b s}}
$$

where $P_{\text {eff }}$ is permeability, $R$ is intestinal radius, as $T_{\mathrm{si}}$ is intestinal transit time, and $T_{\text {abs }}$ is absorption time.

Thus, for a drug to be completely absorbed, it must present $D_{\mathrm{o}}<1, D_{\mathrm{n}}>1$, and $A_{\mathrm{n}}>1$. From these definitions, it is clear that solubility and permeability are the key parameters for absorption control, and in the BCS, drugs are divided into four classes (Figure 2) according to their solubility (high/low) and permeability parameters $(6,7,22)$.

For a drug to be considered highly soluble, its highest dose must be soluble in a volume equal to or less than $250 \mathrm{~mL}$ of an aqueous medium, at a $\mathrm{pH}$ between 1.0 and 7.5. The estimated volume of $250 \mathrm{~mL}$ is based on the protocols of bioequivalence studies that prescribe the administration of the medication to fasting volunteers with one glass of water $(11,18)$.

The classification of drugs based on permeability is based indirectly on the extent of absorption in humans, through the evaluation of the fraction of the dose 


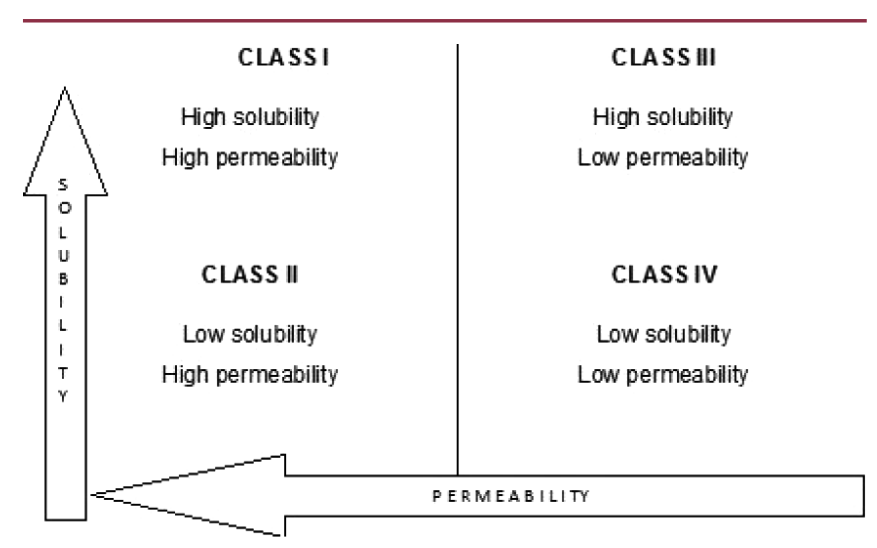

Figure 2. Divisions of the Biopharmaceutics Classification System.

absorbed or directly by measuring the rate of mass transfer across the human intestinal membrane or epithelial membranes. The drug is considered highly permeable when at least $90 \%$ of the administered dose is absorbed $(11,18)$.

Accordingly, drugs may be divided into four groups according to their solubility and permeability (Figure 2), each of which presents special characteristics $(7,9,23)$.

Class I: Drugs in this class have good permeability unless they are unstable in the gastrointestinal tract or if they undergo first-pass metabolism. Because they also have good solubility, the limiting factor for their absorption is the gastric emptying time. Immediate-release solid dosage forms that contain drugs with these characteristics are potential candidates for biowaivers from bioequivalence studies.

Class II: Despite having good permeability, the drugs classified in this group present solubility issues, thus dissolution becomes a limiting factor in absorption. The use of well-defined formulations or pharmaceutical techniques, such as complexation with cyclodextrins or reduction in particle size, among others, may facilitate dissolution and consequently increase oral bioavailability. However, in vitro dissolution tests should mirror the in vivo dissolution properties perfectly.

Class III: This comprises drugs with good solubility and reduced permeability; the latter is considered a limiting factor for absorption. It is important that the dosage forms that contain this type of drug release it quickly to maximize contact time with the intestinal epithelium; however, absorption may be affected by the influence of physiological variables, such as intestinal residence time and luminal content.

Class IV: This is the class that researchers find most challenging, because these drugs present oral bioavailability issues due to reduced solubility and permeability.

\section{Methods Used to Determine Drug Solubility and Permeability \\ Solubility \\ Solubility evaluation for the biopharmaceutical} classification of a substance is based on a determination of the equilibrium under conditions of physiological $\mathrm{pH}$. The FDA suggests that the test be carried out at a temperature of $37 \pm 1^{\circ} \mathrm{C}$ in aqueous media, varying the $\mathrm{pH}$ from 1 to 7.5. The traditional phase solubility method is the most recommended, although it may be replaced by another (e.g., titration) as long as it is capable of providing the solubility equilibrium of the drug $(6,11,18)$.

\section{Permeability}

There are different methods that can be used to classify substances according to permeability, and some of them involve studying pharmacokinetic parameters in humans and determining the permeability of the gastrointestinal tract (11).

According to the FDA, there are several methods for studying pharmacokinetic parameters including a study of mass balance using stable isotopes or marker substances and a study of absolute bioavailability, with the absorption of the drug evaluated in comparison with the absorption of a reference substance. Examples of intestinal permeability methods include intestinal perfusion studies in humans, in vivo or in situ intestinal perfusion studies in animal subjects, in vitro permeation studies on intestinal tissue from humans or animals, and in vitro permeation studies on epithelial cell monolayers (11).

Although pharmacokinetic studies in humans are the gold standard for determining permeability, their use in the development stage is not feasible, mainly because of the high rate of variability, the necessity for administration by a formulation, and the costs involved. Accordingly, intestinal permeability studies have become an interesting alternative (6).

In vitro techniques based on the use of cell cultures have been widely investigated in recent years. Among these cell types, those of the Caco-2 line are the most widely used because of their similarity with intestinal cells and for the capacity of expression of transporters and intestinal enzymes $(6,24)$.

\section{Extension of the BCS}

Since it was created, the Biopharmaceutics Classification System has been the focus of several studies and discussions within the scientific community, and several suggestions have been proposed in an attempt to make classification less restrictive and more broadly applicable (18).

Regarding solubility, alteration of the $\mathrm{pH}$ evaluation range from 1.0-7.5 to 1.0-6.8 has been suggested, considering that $\mathrm{pH} 7.5$ corresponds to the region of the ileum where the drug arrives already dissolved, along with a volume alteration from 250 to $500 \mathrm{~mL}$, which is an 
average because intestinal volume can vary from 50 to $1100 \mathrm{~mL}(18,25)$.

An alteration of the permeability value from $90 \%$ to $85 \%$ has been suggested for highly permeable drugs, because through the evaluation of different drugs considered to have high absorption, the fraction of the absorbed dose determined experimentally has proved to be less than $90 \%(18,25)$.

Besides these suggestions, new methods for the classification of substances have been proposed, such as an intrinsic dissolution test for evaluating solubility and the use of new cell and membrane types for evaluating intestinal permeability, as well as the use of computational or in silico methods $(18,26,27)$.

Although such suggestions have been the subject of much discussion among researchers, the FDA still considers the system as it was initially proposed, while the World Health Organization (WHO) has already approved new evaluation criteria $(25,28,29)$.

\section{INTRINSIC DISSOLUTION}

Skinner and Kanfer (30) suggested that the main physicochemical aspects pertaining to drug absorption are the intrinsic dissolution rate and solubility. Because these two parameters are highly dependent on $\mathrm{pH}$, their influence on absorption could easily be determined by the entire $\mathrm{pH}$ range of the gastrointestinal tract.

Intrinsic dissolution is the dissolution of a pure active substance, and the determination of the dissolution rate can be important during the development of new molecules, because with small quantities of material, it is possible to execute the test and predict potential problems $(13,17,31,32)$.

Accordingly, the intrinsic dissolution rate (IDR) is defined as the dissolution rate of a pure active substance, where the conditions of surface area, temperature, agitation, and medium $\mathrm{pH}$ and ionic strength are all constant. Thus, it is possible to obtain data on the chemical purity and equivalence of drugs from different sources. This information is related to the variability of raw material available on the market, which results from distinctive synthesis processes, especially in the final stages of crystallization, and may lead to different particle sizes, degrees of hydration, habits, and crystalline forms for a single drug $(16,19,32,33)$.

The IDR can be obtained by employing a specific device for this purpose, where the compressed drug is exposed in a dissolution medium over a constant surface area, and its value is expressed in $\mathrm{mg} \mathrm{cm}^{-2} \mathrm{~s}^{-1}(17,34-36)$.

Applications for intrinsic dissolution tests are related to their use as a tool in the characterization of solid-state drugs, such as the determination of thermodynamic parameters associated with transition from crystalline phases, degrees of hydration, the investigation of the phenomenon of mass transfer in the dissolution process, the evaluation of the dissolution rate of a drug in different media (variation of $\mathrm{pH}$ or use of surfactants), and the relationship between the dissolution rate of an active substance and that of its crystalline form $(15,37)$.

Recent studies have proved the usefulness of the IDR in determining solubility in the sphere of the Biopharmaceutics Classification System. Because this test is not related to equilibrium but rather to the rate, there is expected to be a greater correlation in the in vivo dissolution dynamic than in the solubility test. In a conventional solubility test, where a quantity of a drug is kept under constant agitation and temperature until the solution is saturated, any determination of the actual solubility of the material may be compromised because of possible occurrences of recrystallization, which may result in alteration of the crystalline form, and hydrate and solvate formation $(15,18,19,27)$.

Changes on the material surface of the compressed drug during the intrinsic dissolution test may also occur, such as the conversion of amorphous atorvastatin into crystalline atorvastatin (38), the transformation of diclofenac salt into its acid form (37), the hydration of anhydrous forms of carbamazepine and theophylline $(16,33)$, and the conversion of rifampicin into a more stable polymorph (39).

However, because the intrinsic dissolution rate is determined by the correlation between the results of dissolved mass per unit area and collection time, from the profile analysis obtained (presence of positive or negative curvature), it is possible to monitor the test and determine solubility before these transformations occur $(15,17)$.

Another significantly beneficial aspect of intrinsic dissolution is the use of small quantities of drug, which facilitates its use in the evaluation of solubility during the preformulation state (new molecules), when minimum quantities of a drug are available for the execution of the tests $(13,40)$.

\section{Apparatus Used to Determine the IDR}

Pharmacopeias list two types of apparatus for the intrinsic dissolution test: a fixed-disk system, described only in the USP, and a rotating-disk system, known as "Wood's apparatus," described in the USP and the European and British pharmacopeias $(17,32,35,41)$.

The rotating-disk system is used most commonly. A good correlation of results is observed compared with the fixed-disk system and even other systems not detailed in pharmacopeias but tested in several studies to determine the intrinsic dissolution rate. The latter include the flowcell system and a miniature apparatus (Mini-IDR) similar to the rotating disk $(32,33,36,42,43)$.

The characteristics of these apparatus (17) include their use in conventional dissolution equipment. They have a cavity for placing the drug, where a press is required for the formation of the compressed drug. The geometry and size of the exposured surface of the drug are known. When placed in the dissolution equipment, the apparatus enable the compressed drug to be exposed in a place of lower hydrodynamic variability. 


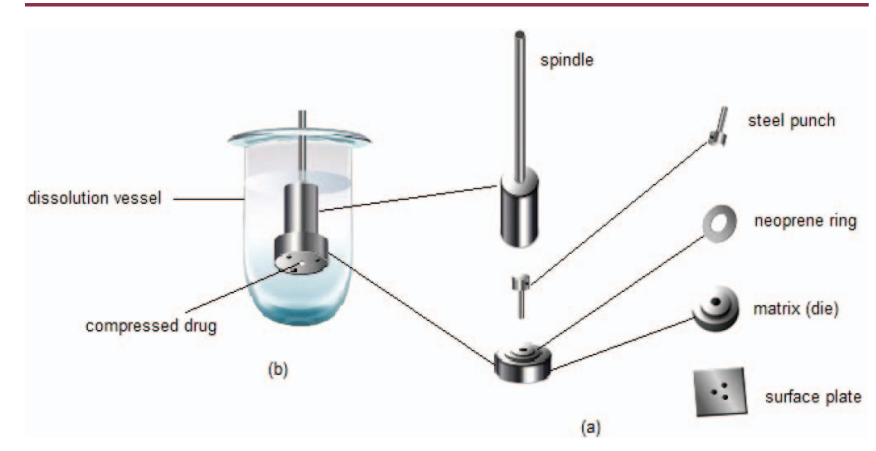

Figure 3. Rotating-disk system (Wood's apparatus): (a) unassembled apparatus and (b) configuration of the method in the dissolution equipment.

The IDR is influenced by various internal and external factors. The internal factors are related to the properties of the solid state of the drug, and the external factors are related to the surface area, the hydrodynamic condition, and the composition of the dissolution medium (viscosity, $\mathrm{pH}$, and ionic strength).

\section{Rotating-Disk System}

This system comprises one steel punch, matrix (die), spindle, neoprene ring, and a surface plate (Figure 3a). The surface plate has three holes to fasten the matrix screws and to aid in the fabrication of the compressed drug. The matrix has a cavity with a diameter of $0.8 \mathrm{~cm}$, where the drug and the punch are inserted and subjected to a compression pressure. Accordingly, after separation from the base, the material is exposed in the face of the matrix, which is coupled to the spindle that controls the movement (Figure 3b) $(17,32)$.

\section{Fixed-Disk System}

The system has a steel punch, matrix, neoprene ring, surface plate, and support (Figure 4a). The compression formation scheme is similar to the rotating-disk apparatus. However, in this case, a support is needed to sustain the matrix and the punch on a vessel with a flat bottom in such a manner that the area of the drug is fixed and exposed upwards, and the rotational movement is controlled by a paddle of Apparatus 2 (Figure 4b) $(17,32$ ).

\section{Calculating IDR and Analyzing Test Data}

To calculate the IDR, an equation derived from the Noyes-Whitney equation is used. For the derivative, two items are removed from the latter equation, the dissolution rate (generally expressed by the dissolved mass of the sample per unit time) and the dissolution flow (when the surface area is taken into account), expressed as dissolution rate per unit area $(15,17,44)$

$$
j=\frac{V d c}{d t} \times \frac{1}{A}
$$

where $j$ is dissolution flow $\left(\mathrm{mg} \mathrm{cm}^{-2} \mathrm{~s}^{-1}\right), V$ is the volume of the dissolution medium $(\mathrm{mL}), c$ is the concentration of

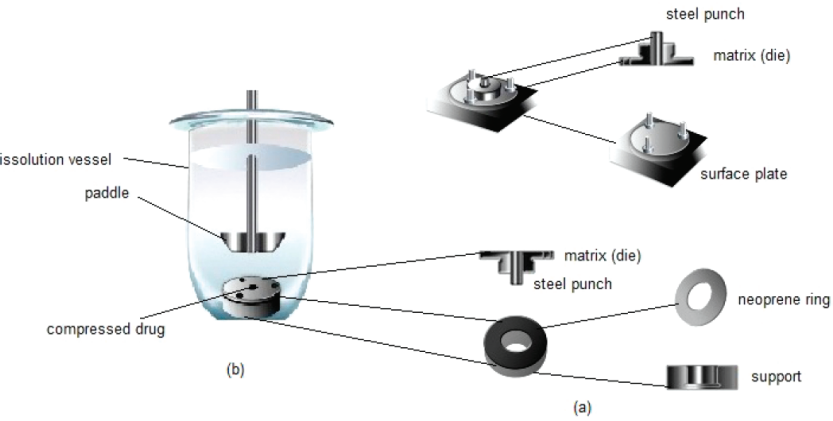

Figure 4. Fixed disk method: (a) unassembled apparatus and (b) configuration of the method in the dissolution equipment.

dissolved drug in the medium $(\mathrm{mg} / \mathrm{mL}), A$ is the surface area of the sample $\left(\mathrm{cm}^{2}\right)$, and $t$ is time $(\mathrm{s})$.

Thus, when the dissolution flow $(j)$ calculation is used for a pure drug, it is more commonly called the intrinsic dissolution rate (17).

During the test, the cumulative quantity of dissolved drug at each time interval must be corrected by accounting for the sampling volumes taken for analysis. For the calculation of the IDR, a graph is constructed of the accumulated amount of dissolved drug as a function of time, and a linear regression is performed. With the attainment of a linear equation, the dissolution rate in units of mass per second is determined, represented by the value of the angular coefficient. The intrinsic dissolution rate is determined by dividing this value by the surface area of the compressed drug $\left(\mathrm{cm}^{2}\right)$ and is reported in units of mass $\mathrm{cm}^{-2} \mathrm{~s}^{-1}(17)$. It is worth remembering that most of the intrinsic dissolution results found in the literature are expressed in $\mathrm{mg} \mathrm{min}^{-1} \mathrm{~cm}^{-2}$, the format used by many authors and recommended by the USP until 2008.

The graph obtained for percent dissolved drug as a function of time may present curvature, and in this case, only the initial linear region must be considered for calculating the dissolution rate. The presence of positive curvature may indicate some experimental problem, while negative curvature may be related to the transition of the crystal form on the surface of the compressed drug or inadvertent achievement of the saturation point of the dissolution medium $(16,17,33)$.

\section{Determination of the Solubility Class from the IDR}

As previously mentioned, studies have been carried out to prove the feasibility of the use of the intrinsic dissolution rate to determine solubility class in the Biopharmaceutics Classification System.

Yu and collaborators (15) determined that for drugs for which it is possible to obtain a good correlation between intrinsic dissolution and solubility, the borderline value between classes is $0.1 \mathrm{mg} \mathrm{min}^{-1} \mathrm{~cm}^{-2} / 0.0017 \mathrm{mg} \mathrm{cm}^{-2} \mathrm{~s}^{-1} . \mathrm{In}$ other words, drugs with an intrinsic dissolution rate above this value would be considered highly soluble, and rates below this limit would indicate drugs with low solubility. 
Table 1. IDR Values ( $\mathrm{mg} \mathrm{min}^{-1} \mathrm{~cm}^{-2}$ ) and BCS Solubility Classification for Several Drugs (Adapted from ref 45.)

\begin{tabular}{lcl}
\hline Drug & IDR $\left(\mathrm{mg} \mathrm{min} \mathrm{cm}^{-2}\right)$ & $\begin{array}{l}\text { BCS Solubility } \\
\text { Classification }\end{array}$ \\
\hline ranitidine & $43.1 \pm 0.1$ & High \\
\hline metoprolol & $27.0 \pm 0.4$ & High \\
\hline propranolol & $17.8 \pm 0.3$ & High \\
\hline nortriptyline & $6.5 \pm 0.2$ & High \\
\hline atenolol & $2.56 \pm 0.13$ & High \\
\hline nadolol & $1.44 \pm 0.04$ & High \\
\hline labetolol & $0.70 \pm 0.02$ & Low \\
\hline cetoprofen & $0.57 \pm 0.03$ & Low \\
\hline furosemide & $0.50 \pm 0.02$ & Low \\
\hline naproxen & $0.26 \pm 0.02$ & Low \\
\hline hydrochlorothiazide & $0.11 \pm 0.01$ & Low \\
\hline piroxicam & $0.088 \pm 0.002$ & Low \\
\hline carbamazepine & $0.029 \pm 0.002$ & Low \\
\hline griseofulvin & $0.0022 \pm 0.0002$ & \\
\hline & & Low \\
\hline
\end{tabular}

Recently, in a study carried out with the same drugs under the same test conditions (rotating system at $100 \mathrm{rpm}$, $900 \mathrm{~mL}$ of $\mathrm{pH} 6.8$ phosphate buffer, Table 1) employed by Yu et al. (45), Zakeri-Milani et al. (19) suggested a value of $1 \mathrm{mg} \mathrm{min}^{-1} \mathrm{~cm}^{-2} / 0.017 \mathrm{mg} \mathrm{cm}^{-2} \mathrm{~s}^{-1}$ as a basis for the division of solubility classes.

Table 2. Conditions and Factors Related to the Solubility Tests by the Phase Solubility Method and Intrinsic Dissolution

\begin{tabular}{|c|c|c|}
\hline Conditions & Solubility & $\begin{array}{c}\text { Intrinsic } \\
\text { Dissolution }\end{array}$ \\
\hline Equipment & Shaker (Incubator) & $\begin{array}{l}\text { Dissolution } \\
\text { equipment }\end{array}$ \\
\hline Apparatus & Flasks & Fixed/rotating disk \\
\hline Quantity of material & $\begin{array}{l}\text { Necessary for } \\
\text { saturation }\end{array}$ & $100-200 \mathrm{mg}$ \\
\hline Time & $\begin{array}{l}\text { Up to } 72 \mathrm{~h} \text { (until } \\
\text { saturation) }\end{array}$ & $\begin{array}{l}30 \text { min to } 24 \mathrm{~h} \text { until } \\
\text { a straight line is } \\
\text { obtained }\end{array}$ \\
\hline $\begin{array}{l}\text { Influence of a reduction in } \\
\text { temperature }\end{array}$ & Yes & No \\
\hline $\begin{array}{l}\text { Filtration or centrifugation } \\
\text { required }\end{array}$ & Yes & No \\
\hline $\begin{array}{l}\text { Possibility to obtain results } \\
\text { before a transition into the } \\
\text { crystalline form of the sample }\end{array}$ & No & Yes \\
\hline
\end{tabular}

Although the limits indicated in the studies are different, probably due to differences in the apparatus used, the order of classification of the evaluated substances was the same. Accordingly, for drugs that do not present very elevated or very low doses, where there are no discrepancies between the solubility and intrinsic dissolution results, the IDR may be considered as a technique to classify solubility $(15,19)$.

\section{SOLUBILITY VERSUS INSTRINSIC DISSOLUTION}

Because intrinsic dissolution is a feasible alternative for determining the solubility class, it can be noted that, as presented in Table 2, this test has several advantages over the phase solubility method, especially with respect to time, quantity of material, and handling of samples.

\section{CONCLUSION}

With the use of the BCS in the evaluation of solubility and permeability characteristics of drugs, it is possible to predict the in vivo behavior of substances and thus avoid any formulation issues and unnecessary expense with bioequivalence studies. Among the methods used for classification, the attainment of the intrinsic dissolution rate is a technique that has been gaining prominence, where with minimal amounts of drug and time, the solubility of substances can be more precisely determined.

\section{REFERENCES}

1. Zhu, H.; Zhou, H.; Seitz, K. Bioavailability and Bioequivalence. In Developing Solid Oral Dosage Forms: Pharmaceutical Theory and Practice; Qiu, Y., Chen, Y., Zhang, G. G. Z., Liu, L., Porter, W. R., Eds.; Academic Press: Burlington, MA, 2009; pp 341-364.

2. Dokoumetzidis, A.; Macheras, P. A century of dissolution research: From Noyes and Whitney to the biopharmaceutics classification system. Int. J. Pharm. 2006, 321, 1-11.

3. Panchagnula, R.; Thomas, N. S. Biopharmaceutics and pharmacokinetics in drug research. Int. J. Pharm. 2000, 201, 131-150.

4. Pelkonen, O.; Boobis, A. R.; Gundert-Remy, U. In vitro prediction of gastrointestinal absorption and bioavailability: an experts' meeting report. Eur.J.Clin. Pharmacol. 2001, 57 (9), 621-629.

5. Lindenberg, M.; Kopp, S.; Dressman, J. B. Classification of orally administered drugs on the World Health Organization model list of essential medicines according to the biopharmaceutics classification system. Eur. J. Pharm. Biopharm. 2004, 58, 265-278.

6. Varma, M.V.S.; Khandavilli, S.; Ashokraj, Y.; Jain, A.; Dhanikula, A.; Sood, A.; Thomas, N. S.; Pillai, O.; Sharma, P.; Gandhi, R.; Agrawal, S.; Nair, V.; Panchagnula, R. Biopharmaceutic classification system: a scientific framework for pharmacokinetic optimization in drug research. Curr. Drug Metab. 2004, 5, 375-388.

7. Amidon, G. L.; Lennernäs, H.; Shah, V. P.; Crison, J.R. A theoretical basis for a biopharmaceutic drug 
classification: The correlation of in vitro drug product dissolution and in vivo bioavailability. Pharm. Res. 1995, 12 (3), 413-420.

8. Lennernäs, H.; Abrahamsson, B. The use of biopharmaceutic classification of drugs in drug discovery and development: current status and future extension. J. Pharm. Pharmacol. 2005, 57, 273-285.

9. Lipka, E.; Amidon, G. L. Setting bioequivalence requirements for drug development based on preclinical data: optimizing oral drug delivery systems. J. Controlled Release 1999, 62, 41-49.

10. Löbenberg, R.; Amidon, G. L. Modern biovailability, bioequivalence and biopharmaceutics classification system. New scientific approaches to international regulatory standards. Eur. J. Pharm. Biopharm. 2000, 50, 3-12.

11. Waiver of in vivo bioavailability and bioequivalence studies for immediate-release solid oral dosage forms based on a biopharmaceutics classification system, Guidance for Industry; U.S.. Department of Health and Human Services. Food and Drug Administration, Center for Drug Evaluation and Research (CDER), U.S. Government Printing Office:Washington, DC, 2000.

12. Rao, V. M.; Sanghvi, R.; Zhu, H. Solubility of Pharmaceutical Solids. In Developing Solid Oral Dosage Forms: Pharmaceutical Theory and Practice; Qiu, Y., Chen, Y., Zhang, G. G. Z., Liu, L., Porter, W. R., Eds.; Academic Press: Burlington, MA, 2009; pp 3-23.

13. Steele, G. Preformulation Predictions from Small Amounts of Compound as an Aid to Candidate Drug Selection. In Pharmaceutical Preformulation and Formulation: A Practical Guide from Candidate Drug Selection to Commercial Dosage Form; Gibson, M., Ed.; Interpharm/CRC: Boca Raton, FL, 2001; pp 21-88.

14. Baka, E.; Comer, J.E.;Takács-Novák, K. Study of equilibrium solubility measurement by saturation shake-flask method using hydrochlorothiazide as model compound. J. Pharm. Biomed. Anal. 2008, 46 (2), 335-341.

15. Yu, L. X.; Carlin, A. S.; Amidon, G. L.; Hussain A. S. Feasibility studies of utilizing disk intrinsic dissolution rate to classify drugs. Int. J.Pharm. 2004, 270 (1-2), 221-227.

16. Sehić, S.; Betz, G.; Hadzidedić, S.; El-Arini, S. K.; Leuenberger, $\mathrm{H}$. Investigation of intrinsic dissolution behavior of different carbamazepine samples. Int. J. Pharm. 2010, 386 (1-2), 77-90.

17. The United States Pharmacopeia and National Formulary USP 32-NF 27; The United States Pharmacopeial Convention, Inc.: Rockville, MD, 2009.

18. Yu, L. X.; Amidon, G. L.; Polli, J. E.; Zhao, H.; Mehta, M. U.; Conner, D. P.; Shah, V. P.; Lesko, L. J.; Chen, M.; Lee, V.H. L.; Hussain, A. S. Biopharmaceutics classification system: The scientific basis for biowaiver extensions. Pharm. Res. 2002, 19 (7), 921-925.

19. Zakeri-Milani, P.; Barzegar-Jalali, M.; Azimi, M.; Valizadeh, H. Biopharmaceutical classification of drugs using intrinsic dissolution rate (IDR) and rat intestinal permeability. Eur.J.Pharm. Biopharm. 2009, 73 (1), 102-106.

20. Ku, M. S. Use of the Biopharmaceutical Classification System in Early Drug Development. AAPS J. 2008, 10 (1), 208-212.

21. Karalis, V.; Macheras, P.; Van Peer, A.; Shah, V.P. Bioavailability and Bioequivalence: Focus on Physiological Factors and Variability. Pharm. Res. 2008, 25 (8), 1956-1962.

22. Martinez, M. N.; Amidon, G. L. A Mechanistic approach to understanding the factors affecting drug absorption: A review of fundamentals. J. Clin. Pharmacol. 2002, $42(6), 620-643$.

23. Ashford, M. Assessment of Biopharmaceutical Properties. In Pharmaceutics: The Science of Dosage Form Design; Aulton, M. E., Ed.; Churchill Livingstone: London, 2002; pp 253-274.

24. Jung, S. J.; Choi, S. O.; Um, S. Y.; Kim, J. I.; Choo, H. Y. P.; Choi, S. Y.; Chung, S.Y. Prediction of the permeability of drugs through study on quantitative structurepermeability relationship. J. Pharm. Biomed. Anal. 2006, 41 (2), 469-475.

25. Proposal to waive in vivo bioequivalence requirements for WHO model list of essential medicines immediaterelease, solid oral dosage forms. WHO technical Report series No. 937; World Health Organization: Geneva, 2006; pp 391-415.

26. Dokoumetzidis, A.; Valsami, G.; Macheras, P. Modelling and simulation in drug absorption processes. Xenobiotica 2007, 37 (10-11), 1052-1065.

27. Yazdanian, M.; Briggs, K.; Jankovsky, C.; Hawi, A.The "high solubility" definition of the current FDA Guidance on Biopharmaceutical Classification System may be too strict for acidic drugs. Pharm. Res. 2004, 21 (2), 293-299.

28. Benet, L. Z.; Amidon, G. L.; Barends, D. M.; Lennernäs, H.; Polli, J. E.; Shah, V. P.; Stavchansky, S. A.; Yu, L. X. The use of BDDCS in classifying the permeability of marketed drugs. Pharm. Res. 2008, 25 (3), 483-488.

29. Papadopoulou, V.; Valsami, G.; Dokoumetzidis, A.; Macheras, P. Biopharmaceutics classification systems for new molecular entities (BCS-NMEs) and marketed drugs (BCD-MD):Theoretical basis and practical examples. Int. J. Pharm. 2008, 361 (1-2), 70-77.

30. Skinner, M.; Kanfer, I. Intrinsic dissolution rate and solubility studies on josamycin, a macrolide antibiotic. Int. J. Pharm. 1992, 88 (1-3), 151-158.

31. Ayres, C.;Burke,W.; Dickinson, P; Kirk, G.;Pugh R.; SharmaSingh, G.; Kittlety, R. Intrinsic dissolution rate determinations in early development and relevance to in vivo performance. Am. Pharm. Rev. 2007, 10 (1), 74-78.

32. Viegas, T. X.; Curatella, R. U.;Winkle, L. L. V.; Brinker, G., Measurement of Intrinsic Drug Dissolution Rates Using Two Types of Apparatus. Pharm. Technol. 2001, 25 (6), 44-53.

33. Lehto, P.; Aaltonen, J.; Niemelä, P.; Rantanen, J.; Hirvonen, J.; Tanninen, V. P.; Peltonen, L. Simultaneous 
measurement of liquid-phase and solid-phase transformation kinetics in rotating disc and channel flow cell dissolution devices. Int.J. Pharm. 2008, $363(1-2), 66-72$.

34. Azarmi, S.; Roa, W.; Löbenberg, R. Current perspectives in dissolution testing of conventional and novel dosage forms. Int. J. Pharm. 2007, 328 (1), 12-21.

35. European Pharmacopoeia, 6th ed.; European Directorate for the Quality of Medicines, Council of Europe: Strasbourg, France, 2008.

36. Peltonen, L.; Liljeroth, P.; Heikkilä, T.; Kontturi, K.; Hirvonen, J. Dissolution testing of acetylsalicylic acid by a channel flow method-correlation to USP basket and intrinsic dissolution methods. Eur. J. Pharm. Sci. 2003, 19, 395-401.

37. Bartolomei, M.; Bertocchi, P.; Antoniella, E; Rodomonte A. Physico-chemical characterisation and intrinsic dissolution studies of a new hydrate form of diclofenac sodium: comparison with anhydrous form. J. Pharm. Biomed. Anal. 2006, 40 (5), 1105-1113.

38. Kim, M.; Jin, S.; Kim, J.; Park, H. J.; Song, H.; Neubert, R. H. $\mathrm{H}$., Hwang, S. Preparation, characterization and in vivo evaluation of amorphous atorvastatin calcium nanoparticles using supercritical antisolvent (SAS) process. Eur. J. Pharm. Biopharm. 2008, 69 (2), 454-465.

39. Agrawal, S.; Ashokraj, Y.; Bharatam, P.; Pillai, O.; Panchagnula, R. Solid-state characterization of rifampicin samples and its biopharmaceutic relevance. Eur.J.Pharm. Sci. 2004, 22 (2-3), 127-144.

40. Wang, J.; Flanagan, D. R. Fundamentals of Dissolution. In Developing Solid Oral Dosage Forms: Pharmaceutical Theory and Practice; Yihong, Q., Chen, Y., Zhang, G. Z.Z., Liu, L., Porter, W., Eds.; Academic Press: Burlington, MA, 2009; pp 309-316.

41. The British Pharmacopoeia, The Stationary Office: London, 2009; pp A310-A311.

42. Berge, C. M.; Tsinman, O.; Voloboy, D.; Lipp, D.; Stones, S.; Avdeef, A. Miniaturized Intrinsic Dissolution Rate (Mini-IDR ${ }^{\mathrm{TM}}$ ) Measurement of Griseofulvin and Carbamazepine.Dissolution Technol. 2007, 14 (4), 39-41.

43. Tsinman, K.; Avdeef, A.; Tsinman, O.; Voloboy, D. Powder Dissolution Method for Estimating Rotating Disk Intrinsic Dissolution Rates of Low Solubility Drugs. Pharm. Res. 2009, 26 (9), 2093-2100.

44. Carstensen, J.T. Dissolution from Particles and Surfaces. In Advanced Pharmaceutical Solids; Drugs and the Pharmaceutical Sciences, Vol. 110; Marcel Dekker, Inc.: New York, 2001; pp 191-194.

45. Yu, L. X.; Carlin A. S.; Hussain, A. S. Feasibility Studies of Intrinsic Dissolution Rate as an Alternative Method to Determine BCS Solubility Membership. Presented at American Association of Pharmaceutical Sciences annual meeting, 2000.

\title{
Corrigendum to "Feasibility Study on Qualification of USP Dissolution Apparatus 1 and 2 Using the Enhanced Mechanical Calibration Procedure"
}

\author{
Brian Yan, Xujin Lu, and Ruben Lozano \\ Analytical \& Bioanalytical Development, Bristol-Myers Squibb Company, \\ New Brunswick, NJ 08903-0191
}

Dissolution Technologies 2011, 18 (2), 17-23.

W ith regard to our article,"Feasibility Study on Qualification of USP Dissolution Apparatus 1 and 2 Using the Enhanced Mechanical Calibration Procedure," which appeared in Dissolution Technologies, Vol. 18, Issue 2, pp 17-23 (May 2011), the authors wish to issue a correction concerning statements that we attributed to the VK7000 dissolution test system. We inaccurately stated in several instances that this model "lacks the feature of levelness adjustment," when in fact, it does possess that feature, thus allowing the unit levelness to be adjusted properly, if necessary. We apologize to Agilent for the oversight. It was unintended for our paper to focus on a comparison of systems or to point out deficiencies. Rather, the main objective was to demonstrate the relationship between system levelness and shaft verticality and its potential impact on meeting the mechanical calibration criteria.

Also, regarding use of the digital device to measure system levelness and shaft verticality, the authors wish to clarify that the bench surface was confirmed to be level relative to the earth (i.e., the lab floor), thereby justifying use of the bench surface as the zero reference point for the digital device. To ensure that digitally recorded measurements of system levelness and shaft verticality are accurate, it is important not to assume automatically that the lab bench is vertical relative to earth level (true zero). 